Pacific Journal of Mathematic 


\title{
ON ULAM'S CONJECTURE FOR SEPARABLE GRAPHS
}

\section{J. A. BONDY}

\begin{abstract}
Ulam's conjecture, that every graph of order greater than two is determined up to isomorphism by its collection of maximal subgraphs, is verified for the case of separable graphs which have no pendant vertices. Partial results are then obtained for the case of graphs with pendant vertices.
\end{abstract}

Unless otherwise stated, the graphs dealt with in this paper will be finite and undirected, and may have loops and multiple edges. Any definitions and notation not given below can be found in Berge [1].

A part $G^{1}$ of a graph $G$ is a subset of the vertices and edges of $G$. The end-vertices of edges in $G^{1}$ need not themselves be in $G^{1}$. If $G^{1}$ is a part of $G, G-G^{1}$ denotes that partial subgraph of $G$ which is obtained by deleting $G^{1}$ and all edges of $G$ which are joined to vertices of $G^{1}$. Now let $S$ be some distinguished set of parts of a graph, and let $S(X)=\left\{X^{i}\right\}$ be the labelled set of these parts in the graph $X$. We call two graphs $G, H$ S-equivalent if $|\boldsymbol{S}(G)|=|\boldsymbol{S}(H)|=$ $M(<\infty)$ and, possibly after relabelling, $G-G^{i} \cong H-H^{i}(1 \leqq i \leqq M)$. $G^{i}, H^{i}$ will be referred to as corresponding parts.

In [8] Ulam proposed the following conjecture.

ConjeCture A. Vertex-equivalent graphs of order greater than two are isomorphic.

Kelly [7] verified this conjecture for trees and, by exhaustion, for all graphs up to order seven. A related conjecture, intuitively simpler but also as yet unsolved, was suggested by Harary [4].

ConjeCtuRE B. Edge-equivalent graphs with more than three edges are isomorphic.

Harary and Palmer [5] strengthened Kelly's theorem by showing that pendant vertex-equivalent trees are isomorphic, and hence proved as corollaries both Conjectures $\mathrm{A}$ and $\mathrm{B}$ for trees. In [2] the author took this one stage further and showed that peripheral vertex-equivalent trees are isomorphic. For directed graphs, Harary and Palmer [5, 6] have proved that vertex-equivalent weak tournaments of order greater than four are isomorphic, that edge-equivalent tournaments are isomorphic, and that pendant vertex-equivalent directed trees with at least three pendant vertices are isomorphic. In [7] Kelly also stated 
that Conjecture A holds for disconnected graphs. A proof is given by Harary in [4]. We here examine this conjecture for graphs of connectivity one.

2. Separable graphs with no pendant vertices. Throughout this section $G, H$ will be taken to be vertex-equivalent graphs of order $N$.

A cut-vertex of a graph is a vertex whose removal disconnects the graph. A connected graph is separable if it contains a cut-vertex. A block is a maximal connected subgraph that is not separable. A pendant vertex is a vertex joined to just one other vertex.

THEOREM 1. Vertex-equivalent separable graphs with no pendant vertices are isomorphic.

We first need two lemmas.

Lemma 1.1. (Kelly [7].) Let $Y$ be any graph of order less than $N$. Suppose there are $\alpha$ distinct subgraphs of $G$ isomorphic to $Y$ and that vertex $u_{i}$ of $G$ is in $\alpha_{i}$ of these subgraphs; that there are $\beta$ distinct subgraphs of $H$ isomorphic to $Y$ and that vertex $v_{i}$ (where $v_{i}$ is the vertex corresponding to $u_{i}$ ) is in $\beta_{i}$ of these subgraphs. Then

$$
\alpha=\beta \text {, and } \alpha_{i}=\beta_{i}(1 \leqq i \leqq N) \text {. }
$$

LEMMA 1.1 remains true if 'subgraph' is replaced by 'partial subgraph' throughout. We shall refer to this version as Lemma 1.1 (a).

Note. It is an easy consequence of Lemma 1.1 that corresponding vertices have the same number of loops and are of the same degree; it is also clear that $G$ and $H$ have the same number of pendant vertices.

Lemma 1.2. Suppose $G$ has blocks $B_{1}, B_{2}, \cdots, B_{m}$ (where $m>1$ ) and $H$ has blocks $C_{1}, C_{2}, \cdots, C_{n}$. Then $m=n$, and the blocks can be relabelled so that $B_{i} \cong C_{i}(1 \leqq i \leqq n)$.

Proof. Let $B_{i}$ have order $b_{i}$ and $C_{i}$ have order $c_{i}$. We may assume that $b_{1} \geqq b_{2} \geqq \cdots \geqq b_{m}$ and $c_{1} \geqq c_{2} \geqq \cdots \geqq c_{n}$. The proof will be by induction. Suppose we have already shown that $B_{1} \cong C_{1}, B_{2} \cong$ $C_{2}, \cdots, B_{k} \cong C_{k}$. Since $G$ and $H$ have the same order, $k=m$ if and only if $k=n$, and in that case $G \cong H$. Otherwise $k<\min (m, n)$ and we may suppose that $b_{k+1} \geqq c_{k+1}$. In Lemma 1.1 take $Y$ to be isomorphic to $B_{k+1}$. If $Y$ occurs $\gamma$ times in $\bigcup_{1}^{k} B_{i}$, then $Y$ also occurs $\gamma$ times in $\mathrm{U}_{1}^{k} C_{i}$. Now $Y$ occurs at least $\gamma+1$ times in $G$, since $Y \cong B_{k+1}$. 
Hence $Y$ occurs at least once in $\mathrm{U}_{k+1}^{n} C_{i}$. Therefore $Y$ is isomorphic to a subgraph of $C_{j}$ for some $j>k$. But

order of $Y=$ order of $B_{k+1}=b_{k+1} \geqq c_{k+1} \geqq c_{j}=$ order of $C_{j}$

and hence $Y \cong C_{j}$. Without loss of generality we may take $j=k+1$. Hence, $B_{k+1} \cong C_{k+1}$. Induction is started by the same argument, with $k=0$. Therefore, $m=n$ and $B_{i} \cong C_{i}$ for all $i$.

Proof of Theorem 1. Let $B_{1}$ be a 'pendant' block of $G$ (that is, a block containing just one cut-vertex of $G$ ) such that no pendant block of $G$ or $H$ has order less than $b_{1}$, the order of $B_{1}$. (The assumption that $B_{1}$ is in $G$ results in no loss of generality.) Let $u$ be the cut-vertex joining $B_{1}$ to the rest of $G$. Write $G_{1}=G-\left(B_{1}-u\right)$ and denote by $G_{1}^{s}$ the graph obtained from $G_{1}$ by adding $s$ isolated vertices and joining each to $u$ by one edge. Then $G_{1}^{1}$ is a proper partial subgraph of $G$ and hence, by Lemma 1.1 (a), there is a partial subgraph $H_{1}^{1}$ of $H$ isomorphic to $G_{1}^{1}$, i.e., $\psi\left(G_{1}^{1}\right)=H_{1}^{1}, \psi(u)=v$, say. (Note: $u$ and $v$ are not necessarily corresponding vertices.) Let $H_{1}$ be the graph obtained from $H_{1}^{1}$ by deleting its pendant vertex. Then it is clear that $H_{1}=\psi\left(G_{1}\right)$ and, by Lemma $1.2, H_{1}$ has one block fewer than $H$, that block, $C_{1}$ say, being isomorphic to $B_{1}$. Now $H$ is obtained from $H_{1}$ by adding $b_{1}-2$ vertices and some edges. Since no pendant block of $H$ has order less than that of $B_{1}$, and since $H$ has no pendant vertices, it is easy to see that those edges can only be incident with $v, p$ (the pendant vertex of $H_{1}^{3}$ ) and the $b_{1}-2$ new vertices. Thus $v$ is a cut-vertex of $H$ and it follows that the subgraph of $H$ on $v, p$, and these $b_{1}-2$ new vertices is isomorphic to $C_{1}$. It now remains to show that there is an isomorphism of $B_{1}$ and $C_{1}$ mapping $u$ onto $v$. Denote by $B_{1}^{1}$ the graph obtained from $B_{1}$ by adding an isolated vertex and joining it to $u$ by one edge. Define $C_{1}^{1}$ analogously. It will suffice to prove that $B_{1}^{1} \cong C_{1}^{1}$.

By Lemma 1.1, $G$ and $H$ have the same number of cut-vertices of the same degree, and hence $u$ and $v$ have the same degree, say $r+s+2 t$, where $t$ is the common number of loops at $u$ and $v$. Suppose that, apart from loops, $r$ edges of $G_{1}$ and $s$ edges of $B_{1}$ are joined to $u$. Then it is clear that $r$ edges of $H_{1}$, and hence $s$ edges of $C_{1}$, are joined to $v$. If $B_{1}^{1}$ occurs $\alpha$ times in $G_{1}^{s}$, it occurs $\alpha+r$ times in $G$. But then $B_{1}^{\prime}$ occurs $\alpha$ times in $H_{1}^{s}$ (since $G_{1}^{s} \cong H_{1}^{s}$ ) and $\alpha+r$ times in $H$ (by Lemma 1.1). This implies that $B_{1}^{1} \cong C_{1}^{1}$ and hence $G \cong H$.

3. Graphs with pendant vertices. The trunk $T(G)$ of a graph $G$ is that subgraph of $G$ which remains after repeated removal of pendant vertices. A $\operatorname{limb} L$ of $G$ is a nontrivial maximal connected 
subgraph of $G$ having just one vertex in common with $T(G)$. This vertex is called the root of $L . G$ is the union of its trunk and limbs. The situation is illustrated in Figure 1.

Note. It is clear that these definitions only have meaning for graphs containing cycles of length greater than two.
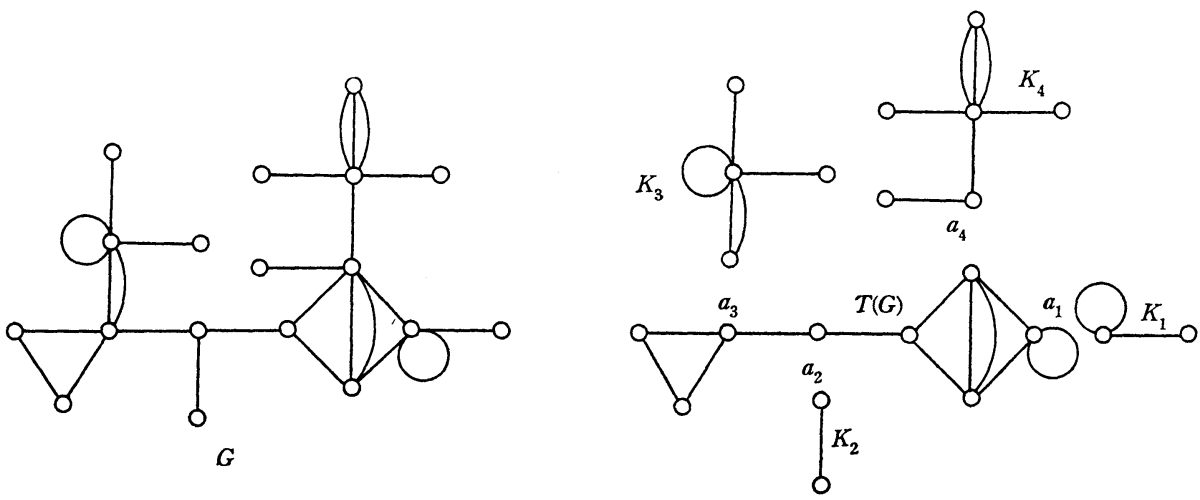

Figure 1.

Suppose $G$ and $H$ are vertex-equivalent graphs. We omit the case when $G$ and $H$ have two blocks, one of which is of order two. This seems to be very difficult to deal with, and is not amenable to the methods used here. If $G$ has only one pendant vertex, on a limb of order greater than two, then $H$ also has these properties and $G \cong H$. For let $u$ be the pendant vertex of $G$, and suppose that there are $l$ loops and $m$ other edges incident with $u$. Then $G$ is obtained from $G-u$ by adding an isolated vertex with $l$ loops, and joining it by $m$ multiple edges to the unique pendant vertex of $G-u$. If vertex $v$ of $H$ corresponds to $u$, then $H$ can be reconstructed from $H-v$ in the same way. Now suppose that $G$ and $H$ have exactly two pendant vertices such that (i) each maximal subgraph of $G$ obtained by the deletion of a pendant vertex has just one limb, of order two; (ii) there is a maximal subgraph of $G$ containing two isolated vertices. Then, by an argument similar to that used above, we again have $G \cong H$. These cases will be excluded from the following discussion.

For the rest of this section we make the weaker assumption that $G, H$ are pendant vertex-equivalent connected graphs. Since Harary and Palmer's theorem for trees extends quite easily to graphs with cycles of length at most two, we consider here only graphs having cycles of length greater than two. It is immediate that $G$ and $H$ have isomorphic trunks. We now look at their limbs. $\theta, \phi, \phi^{\prime}$, respectively will denote isomorphisms of $G-u_{1}$ onto $H-v_{1}, G-u_{2}$ onto $H-v_{2}$ and $G-u_{2}^{\prime}$ onto $H-v_{2}^{\prime}$, where $\left(u_{1}, v_{1}\right),\left(u_{2}, v_{2}\right)$, and $\left(u_{2}^{\prime}, v_{2}^{\prime}\right)$ are pairs 
of corresponding pendant vertices, and $v_{2}^{\prime}=\theta\left(u_{2}\right)$.

Lemma 2.1. Let $G$ and $H$ have limbs $K_{1}, K_{2}, \cdots, K_{m}$, and $L_{1}$, $L_{2}, \cdots, L_{n}$, respectively, where $K_{i}$ has order $k_{i}$ and $L_{i}$ has order $l_{i}$. Assume that $k_{1} \leqq k_{2} \leqq \cdots \leqq k_{m}$, and that $l_{1} \leqq l_{2} \leqq \cdots \leqq l_{n}$. Then $m=n$ and $k_{i}=l_{i}(1 \leqq i \leqq n)$.

Proof. Suppose that $k_{i}=l_{i}(1 \leqq i<r)$ but that $k_{r} \neq l_{r}$, say $k_{r}<l_{r}$. Let $u_{1} \in K_{r}$. If $k_{r}>2$, the combined order of the $r$ smallest limbs in $G-u_{1}$ is $\sum_{i=1}^{r} k_{i}-1$, and the combined order of the $r$ smallest limbs in $H-v_{1}$ is at least $\sum_{i=1}^{r} l_{i}-1>\sum_{i=1}^{r} k_{i}-1$. This contradicts the fact that $\theta\left(G-u_{1}\right)=H-v_{1}$. Therefore $k_{r}=2$ and $l_{r}>2$. Now there is a pendant vertex $u_{2}$ in some $K_{i}(1 \leqq i \leqq r)$ such that $v_{2} \notin L_{j}$ for all $j<r$. Then $G-u_{2}$ has $m-1$ limbs and $H-v_{2}$ has $n$ limbs. Therefore $m=n+1$ and this implies that $l_{i}>2(1 \leqq i \leqq n)$ and that $k_{i}=2(1 \leqq i \leqq n+1)$. Since $G$ and $H$ clearly have the same order

$$
n+1 \sum_{1}^{n+1}\left(k_{i}-1\right)=\sum_{1}^{n}\left(l_{i}-1\right) \geqq 2 n
$$

and therefore $n=1$ and $l_{1}=3$. But this is precisely the case excluded at the beginning of this section.

In the light of Lemma 2.1 we now assume that $G$ and $H$ have limbs $\left\{K_{i}\right\}_{1}^{n}$ and $\left\{L_{i}\right\}_{1}^{n}$ respectively, arranged so that $k_{1} \leqq k_{2} \leqq \cdots \leqq k_{n}$, where $k_{i}$ is the (common) order of $K_{i}$ and $L_{i}$; also that $K_{i}$ has root $a_{i}$ in $T(G)$ and that $L_{i}$ has root $b_{i}$ in $T(H)$. The notation $(U, u) \cong$ $(V, v)$ (or $\alpha(U, u)=(V, v)$ ) will be used to denote that graphs $U$ and $V$ are isomorphic under an isomorphism $(\alpha)$ mapping vertex $u \in U$ onto vertex $v \in V$.

THEOREM 2. The limbs of $G$ and $H$ can be arranged so that $\left(K_{i}, a_{i}\right) \cong\left(L_{i}, b_{i}\right)(1 \leqq i \leqq n)$.

Proof. (a) $n \geqq 2$. Let $u_{1} \in K_{1}, u_{2} \in K_{2}$. Then $G-u_{1}$ has limbs of order $k_{1}-1, k_{2}, \cdots, k_{n}$, and hence so has $H-v_{1}$. We may therefore assume that $v_{1} \in L_{1}$. Then $\theta\left(G-u_{1}\right)=H-v_{1} \Rightarrow$

$$
\theta\left(K_{i}, a_{i}\right)=\left(L_{i}, b_{i}\right) \quad(2 \leqq i \leqq n) .
$$

There are now three subcases:

(i ) $k_{2}>k_{1}+1$. Then $\phi\left(G-u_{2}\right)=H-v_{2} \Rightarrow \phi\left(K_{1}, a_{1}\right)=\left(L_{1}, b_{1}\right)$.

(ii) $k_{2}=k_{1}+1$. From (1), $\theta\left(u_{2}\right)=v_{2}^{\prime} \in L_{2}$ and hence $\theta\left(K_{2}-u_{2}, a_{2}\right)$ $=\left(L_{2}-v_{2}^{\prime}, b_{2}\right) . \quad \phi\left(G-u_{2}\right)=H-v_{2} \Rightarrow$ either $\quad \phi\left(K_{1}, a_{1}\right)=\left(L_{1}, b_{1}\right) \quad$ or $\phi\left(K_{2}-u_{2}, a_{2}\right)=\left(L_{1}, b_{1}\right) . \phi^{\prime}\left(G-u_{2}^{\prime}\right)=H-v_{2}^{\prime} \Rightarrow \operatorname{either} \phi^{\prime}\left(K_{1}, a_{1}\right)=\left(L_{1}, b_{1}\right)$ or $\phi^{\prime}\left(K_{1}, a_{1}\right)=\left(L_{2}-v_{2}^{\prime}, b_{2}\right)$. Therefore at least one of $\phi, \phi^{\prime}, \phi \theta^{-1} \phi^{\prime}$ (product of isomorphisms) maps $\left(K_{1}, a_{1}\right)$ onto $\left(L_{1}, b_{1}\right)$. 
(iii) $k_{2}=k_{1} . \quad$ Suppose $v_{2} \in L_{t} . \quad$ Then $\phi\left(G-u_{2}\right)=H-v_{2} \Rightarrow$

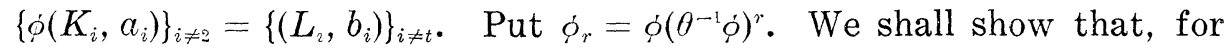
some $r$, either

$$
\phi_{r}\left(K_{1}, a_{1}\right)=\left(L_{1}, b_{1}\right) \text { or } \dot{\phi}_{r}\left(K_{1}, a_{1}\right)=\left(L_{2}, b_{2}\right) .
$$

For assume otherwise. Then $\phi_{r}\left(K_{1}, a_{1}\right)$ is well-defined for all $r$ and we may put $\phi_{r}\left(K_{1}, a_{1}\right)=\left(L_{j_{r}}, b_{j_{r}}\right)$, where $j_{r}>2$. Thus

$$
\theta^{-1} \dot{\phi}_{r}\left(K_{1}, a_{1}\right)=\theta^{-1}\left(L_{j_{r}}, b_{j_{r}}\right)=\left(K_{j_{r}}, a_{\jmath_{r}}\right) \text {. }
$$

Since $n$ is finite there are integers $p, q,(p<q)$ such that $\left(L_{j_{p}}, b_{j_{p}}\right)=$ $\left(L_{j_{q}}, b_{j_{q}}\right)$. Then $\dot{\rho}_{p}\left(K_{1}, a_{1}\right)=\dot{\phi}_{q}\left(K_{1}, a_{1}\right)$, i.e., $\theta^{-1} \dot{\rho}_{q-p-1}\left(K_{1}, a_{1}\right)=\left(K_{1}, a_{1}\right)$, contradicting (3). Hence (2) follows. From (1) $v_{2}^{\prime} \in L_{2}$ and, by an analogous argument to the above, $\phi^{\prime}\left(G-u_{2}^{\prime}\right)=H-v_{2}^{\prime} \Rightarrow$ either $\phi_{s}^{\prime}\left(K_{1}, a_{1}\right)=$ $\left(L_{1}, b_{1}\right)$ or $\dot{\phi}_{s}^{\prime}\left(K_{2}, a_{2}\right)=\left(L_{1}, b_{1}\right)$ for some $s$, where $\phi_{s}^{\prime}=\phi^{\prime}\left(\theta^{-1} \phi^{\prime}\right)^{s}$. Therefore for some $r, s$, at least one of $\phi_{r}, \phi_{s}^{\prime}, \phi_{s}^{\prime} \theta^{-1} \phi_{r}$ maps $\left(K_{1}, a_{1}\right)$ onto $\left(L_{1}, b_{1}\right)$. (i), (ii) and (iii) together imply that an isomorphism $\theta_{1}$ exists such that

$$
\theta_{1}\left(K_{1}, a_{1}\right)=\left(L_{1}, b_{1}\right) .
$$

This completes the proof of part (a).

(b) $n=1$. If $a_{1}$ is not pendant in $K_{1}$, apply the methods of Lemma 2.1 and part (a) of this proof to the sublimbs of $K_{1}$ and $L_{1}$ branching from $a_{1}$ and $b_{1}$ respectively. Otherwise let $a_{1}^{\prime}$ be the first vertex along $K_{1}$ from $a_{1}$ that is joined to more than two other vertices of $K_{1}$ (such a vertex exists since $G$ has at least two pendant vertices). Similarly let $b_{1}^{\prime}$ be the first vertex along $L_{1}$ from $b_{1}$ that is joined to more than two other vertices of $L_{1}$. It is not difficult to see that the subgraph of $K_{1}$ lying between $a_{1}$ and $a_{1}^{\prime}$ is isomorphic to the subgraph of $L_{1}$ lying between $b_{1}$ and $b_{1}^{\prime}$. Now apply the methods of Lemma 2.1 and part (a) of this proof to the sublimbs of $K_{1}$ and $L_{1}$ which branch outwards from $a_{1}^{\prime}$ and $b_{\llcorner}^{\prime}$ respectively.

\section{COROLLARY 2.1. If $k_{1}>2$, then $G \cong H$.}

Proof. Let $u_{1} \in K_{1}$. We may assume that $v_{1} \in L_{1}$. Then $\theta: G-u_{1} \rightarrow$ $H-v_{1}$ induces an isomorphism of $T(G)$ and $T(H)$ mapping $a_{i}$ onto $b_{i}(1 \leqq i \leqq n)$, possibly after some relabeiling. In addition it is clear that $\theta\left(K_{i}, a_{i}\right)=\left(L_{i}, b_{i}\right)(2 \leqq i \leqq n)$, and hence also, by Theorem 2 , that $\left(K_{1}, a_{1}\right) \cong\left(L_{1}, b_{1}\right)$. Therefore $G \cong H$.

In the same way one can prove:

Corollary 2.2. If, for some $i, k_{i+1}-k_{\imath}>1$, then $G \cong H$. 
When there are limbs of order two, one has in general (that is, except for the situation in Corollary 2.2) to deal with a problem concerning the automorphism group of the trunk. Some progress in this direction has been made by Greenwell and Hemminger [3]. However, we have one result which by-passes this difficulty.

CoROLlary 2.3. If the trunk of $G$ is a complete subgraph then $G \cong H$.

Proof. By Theorem $2\left(K_{i}, a_{i}\right) \cong\left(L_{i}, b_{i}\right)(1 \leqq i \leqq n)$. Since $T(G)$ and $T(H)$ are complete, this isomorphism can be extended to an isomorphism of $G$ and $H$ by mapping the vertices of $T(G)$ which are not in the set $\left\{a_{i}\right\}_{1}^{n}$ onto distinct vertices of $T(H)$ not in the set $\left\{b_{i}\right\}_{1}^{n}$.

We conclude by proposing a conjecture analogous to Conjectures $\mathrm{A}$ and $\mathrm{B}$.

Conjecture C. Pendant vertex-equivalent graphs with at least $k$ pendant vertices are isomorphic ( $k$ to be determined).

For this conjecture to be true we must obviously have $k>2$. I am indebted to Dr. Peter M. Neumann for pointing out a counterexample when $k=3$. Figure 2 pictures the two non-isomorphic pendant vertex equivalent graphs.

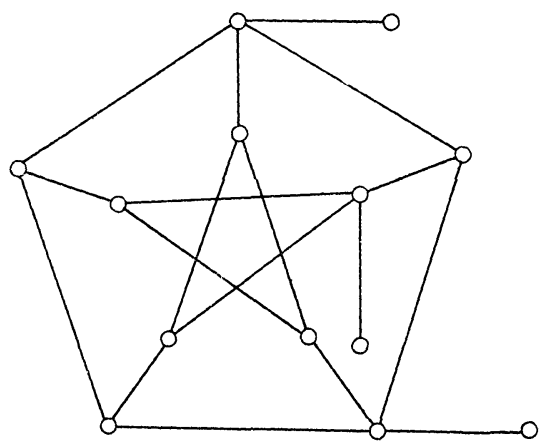

$G$

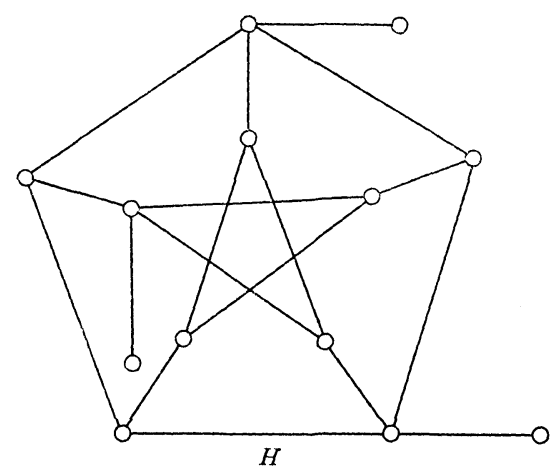

Figure 2.

Dr. Neumann informs me that he has also found a counter-example when $k=4$. 


\section{REFERENCES}

1. C. Berge, Theory of graphs, Dunod, Paris, 1958; reprinted: Methuen, London, 1962.

2. J. A. Bondy, On Kelly's congruence theorem for trees, Proc. Cambridge Philos. Soc. 65 (1969), 387-397.

3. D. L. Greenwell and R. L. Hemminger, Reconstructing graphs.

4. F. Harary, On the reconstruction of a graph from a collection of subgraphs, in Theory of graphs and its applications, proceedings of the symposium held in Smolenice, 1963, 47-52.

5. F. Harary and E. Palmer, The reconstruction of a tree from its maximal subtrees, Canad. J. Math. 18 (1966), 803-810.

6. F. Harary and E. Palmer, On the problem of reconstructing a tournament from subtournaments, Monatsh. Math. 71 (1967), 14-23.

7. P. J. Kelly, A congruence theorem for trees, Pacific J. Math. 7 (1957), 961-968.

8. S. M. Ulam, $A$ Collection of mathematical problems, Wiley, New York, 1960.

Received February 10, 1969.

UNIVERSITY OF WATERLOO

WATERlOO, ONTARIo 


\section{PACIFIC JOURNAL OF MATHEMATICS}

\section{EDITORS}

\author{
H. ROYDEN \\ Stanford University \\ Stanford, California \\ Richard Pierce \\ University of Washington \\ Seattle, Washington 98105
}

\author{
J. DugundJI \\ Department of Mathematics \\ University of Southern California \\ Los Angeles, California 90007 \\ BASIL GoRDON \\ University of California \\ Los Angeles, California 90024
}

\section{ASSOCIATE EDITORS}
E. F. BECKENBACH
B. H. Neumann
F. WOLF
K. YoSHIDA

\section{SUPPORTING INSTITUTIONS}

\author{
UNIVERSITY OF BRITISH COLUMBIA \\ CALIFORNIA INSTITUTE OF TECHNOLOGY \\ UNIVERSITY OF CALIFORNIA \\ MONTANA STATE UNIVERSITY \\ UNIVERSITY OF NEVADA \\ NEW MEXICO STATE UNIVERSITY \\ OREGON STATE UNIVERSITY \\ UNIVERSITY OF OREGON \\ OSAKA UNIVERSITY \\ UNIVERSITY OF SOUTHERN CALIFORNIA
}

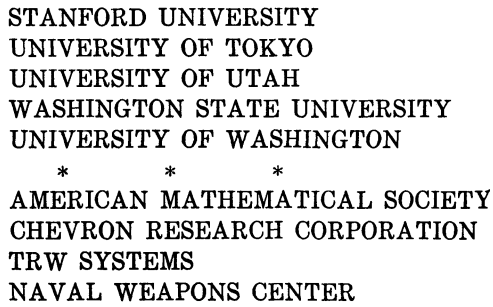

The Supporting Institutions listed above contribute to the cost of publication of this Journal, but they are not owners or publishers and have no responsibility for its content or policies.

Mathematical papers intended for publication in the Pacific Journal of Mathematics should be in typed form or offset-reproduced, double spaced with large margins. Underline Greek letters in red, German in green, and script in blue. The first paragraph or two must be capable of being used separately as a synopsis of the entire paper. It should not contain references to the bibliography. Manuscripts, in duplicate if possible, may be sent to any one of the four editors. Please classify according to the scheme of Math. Rev. 36, 1539-1546. All other communications to the editors should be addressed to the managing editor, Richard Arens, University of California, Los Angeles, California, 90024.

50 reprints are provided free for each article; additional copies may be obtained at cost in multiples of 50 .

The Pacific Journal of Mathematics is published monthly. Effective with Volume 16 the price per volume (3 numbers) is $\$ 8.00$; single issues, $\$ 3.00$. Special price for current issues to individual faculty members of supporting institutions and to individual members of the American Mathematical Society: $\$ 4.00$ per volume; single issues $\$ 1.50$. Back numbers are available.

Subscriptions, orders for back numbers, and changes of address should be sent to Pacific Journal of Mathematics, 103 Highland Boulevard, Berkeley, California, 94708.

PUBLISHED BY PACIFIC JOURNAL OF MATHEMATICS, A NON-PROFIT CORPORATION

Printed at Kokusai Bunken Insatsusha (International Academic Printing Co., Ltd.), 7-17, Fujimi 2-chome, Chiyoda-ku, Tokyo, Japan. 


\section{Pacific Journal of Mathematics}

\section{Vol. 31, No. $2 \quad$ December, 1969}

Efraim Pacillas Armendariz, Quasi-injective modules and stable torsion

classes..........................................

J. Adrian (John) Bondy, On Ulam's conjecture for separable graphs...

Vasily Cateforis and Francis Louis Sandomierski, On commutative rings over which the singular submodule is a direct summand for every module .....

Rafael Van Severen Chacon, Approximation of transformations with continuous

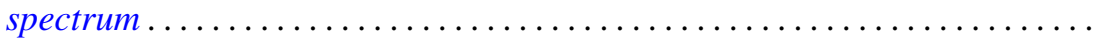

Raymond Frank Dickman and Alan Zame, Functionally compact spaces ...... 303

Ronald George Douglas and Walter Rudin, Approximation by inner

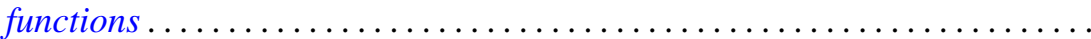

John Walter Duke, A note on the similarity of matrix and its conjugate

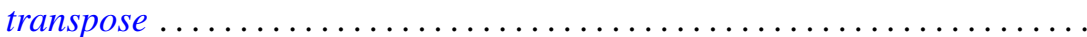

Micheal Neal Dyer and Allan John Sieradski, Coverings of mapping

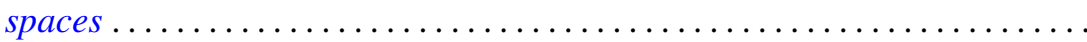

Donald Campbell Dykes, Weakly hypercentral subgroups of finite groups .....

Nancy Dykes, Mappings and realcompact spaces.....................

Edmund H. Feller and Richard Laham Gantos, Completely injective

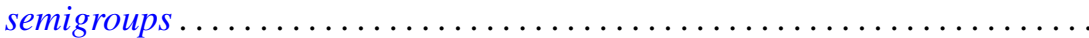

Irving Leonard Glicksberg, Semi-square-summable Fourier-Stieltjes

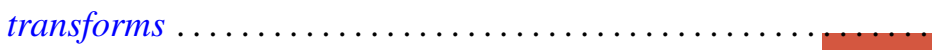

Samuel Irving Goldberg and Kentaro Yano, Integrability of almost cosymplectic structures...

Seymour Haber and Charles Freeman Osgood, On the sum $\sum\langle n \alpha\rangle^{-t}$ and numerical integration ..........................

Sav Roman Harasymiv, Dilations of rapidly decreasing functions ....

William Leonard Harkness and R. Shantaram, Convergence of a sequence of

transformations of distribution functions

Herbert Frederick Kreimer, Jr., A note on the outer Galois theory of rings ...

James Donald Kuelbs, Abstract Wiener spaces and applications to analysis. .

Roland Edwin Larson, Minimal $T_{0}$-spaces and minimal $T_{D}$-spaces...

A. Meir and Ambikeshwar Sharma, On Ilyeff's conjecture .

Isaac Namioka and Robert Ralph Phelps, Tensor products of compact convex sets....

James L. Rovnyak, On the theory of unbounded Toeplitz operators ....

Benjamin L. Schwartz, Infinite self-interchange graphs.......

George Szeto, On the Brauer splitting theorem...

Takayuki Tamura, Semigroups satisfying identity $x y=f(x$,

Kenneth Tolo, Factorizable semigroups .................. 